\title{
Noninvasive 3D Field Mapping of Complex Static Electric Fields
}

\author{
Andreas Kainz, ${ }^{1,}$ Franz Keplinger, ${ }^{1}$ Wilfried Hortschitz, ${ }^{2}$ Matthias Kahr, ${ }^{2}$ Harald Steiner, ${ }^{2}$ Michael Stifter, ${ }^{2}$ \\ James R. Hunt, ${ }^{3,4}$ Javier Resta-Lopez, ${ }^{3,4}$ Volodymyr Rodin, ${ }^{3,4}$ Carsten P. Welsch, ${ }^{3,4}$ Jan Borburgh, ${ }^{5}$ \\ Matthew Alexander Fraser, ${ }^{5}$ and Wolfgang Bartmann ${ }^{5}$ \\ ${ }^{1}$ Institute of Sensor and Actuator Systems, TU Wien, 1040 Vienna, Austria \\ ${ }^{2}$ Department of Integrated Sensor Systems, Danube University Krems, 2700 Wiener Neustadt, Austria \\ ${ }^{3}$ QUASAR Group, The Cockcroft Institute, Daresbury, WA4 4AD, United Kingdom \\ ${ }^{4}$ Department of Physics, University of Liverpool, L69 3BX, United Kingdom \\ ${ }^{5}$ Accelerator Beam Transfer group, CERN, 1211 Geneva, Switzerland
}

(Received 30 January 2019; published 21 June 2019)

\begin{abstract}
Many upcoming experiments in antimatter research require low-energy antiproton beams. With a kinetic energy in the order of $100 \mathrm{keV}$, the standard magnetic components to control and focus the beams become less effective. Therefore, electrostatic components are being developed and installed in transfer lines and storage rings. However, there is no equipment available to precisely map and check the electric field generated by these elements. Instead, one has to trust in simulations and, therefore, depend on tight fabrication tolerances. Here we present, for the first time, a noninvasive way to experimentally probe the electrostatic field in a 3D volume with a microsensor. Using the example of an electrostatic quadrupole focusing component, we find excellent agreement between a simulated and real field. Furthermore, it is shown that the spatial resolution of the probe is limited by the electric field curvature which is almost zero for the quadrupole. With a sensor resolution of $61 \mathrm{~V} / \mathrm{m} / \sqrt{\mathrm{Hz}}$, the field deviation due to a noncompliance with the tolerances can be resolved. We anticipate that this compact and practical field strength probe will be relevant also for other scientific and technological disciplines such as atmospheric electricity or safeguarding near power infrastructure.
\end{abstract}

DOI: 10.1103/PhysRevLett.122.244801

Introduction.-Since the 1950 s it has been commonplace to utilize dedicated quadrupole magnets to focus and control the particle beams contained and accelerated by synchrotrons, linear accelerators, and their corresponding transport lines. Technology has been widely developed to measure these magnetic fields, based on the Hall effect, nuclear magnetic resonance, or coil systems [1-4]. Imperfections in the fabrication process and other sources of error, such as how the effective field strength scales with input current, may be identified. Additionally, fringe fields, higher order field effects, and field imperfections may be studied through such measurements.

With the growth of low-energy antimatter physics and ever lower energy antiproton beams, facilities [5-7] are opting to use electrostatic elements to control such beams. These are more desirable for low-energy hadronic or ion beams as their electrostatic rigidity is independent of mass. This can be seen when considering a charged beam

Published by the American Physical Society under the terms of the Creative Commons Attribution 4.0 International license. Further distribution of this work must maintain attribution to the author(s) and the published article's title, journal citation, and DOI. passing through either a uniform electrostatic or magnetic field:

$$
\rho E=\frac{2 E_{\mathrm{k}}}{q}, \quad \rho B=\frac{1}{q} \sqrt{2 m E_{\mathrm{k}}},
$$

where $\rho$ is the bending radius of the beam, $E$ the electric field, $B$ the magnetic field, $E_{\mathrm{k}}$ the kinetic energy of the beam, $m$ the mass, and $q$ the charge of the particles. For medium to higher energies magnetic elements are preferable due to a dependence on $\sqrt{E_{\mathrm{k}}}$. Despite this advantage for low-energy beams, precise field mapping has never been performed for electrostatic elements due to a lack of appropriate noninvasive field measurement technology. Instead one is relying on a good agreement between simulated and real field and on flawless fabrication of the hardware elements. Currently the extra low energy antiproton (ELENA) storage ring is undergoing commissioning at CERN, decelerating $5.3 \mathrm{MeV}$ beams to $100 \mathrm{keV}$ [7-10]. To accommodate these unprecedented low energies, magnetic transport lines to antimatter experiments are being replaced by electrostatic lines. The field homogeneity requirements are controlled by specifying carefully the mechanical tolerances on electrode shape and alignment. We present an approach for verifying experimentally the 
simulated field using the example of an electrostatic quadrupole (EQP), which is part of a focusing-defocusing assembly (FODO) of these lines. With this approach, we close a long-open gap in measurement technology and show that the field changes due to offsets in the position of the EQP electrodes within the tolerances can be resolved. This way, faulty assemblies can be identified and excluded from application in the transfer lines.

Reliable determination of slowly varying and static electric fields has repeatedly proven to be challenging. Especially the scanning or mapping of $E$ fields in a threedimensional volume has not been achieved with state-ofthe-art equipment. For example, the standard instrument in atmospheric science is the so-called field mill [11-13], which chops the electrostatic field with a grounded electrode. The time-varying induced charge on the electrode is then converted into a voltage signal. This method has been miniaturized to profit from reduced power consumption and size [14-16]. The grounded component, however, causes massive field distortions directly at the measurement point.

Another method employs electro-optical crystals. Here, changes in the refractive index proportional to $E$ (Pockels effect) or to $E^{2}$ (Kerr effect) are used [17-19]. Such sensors, however, are hardly of practical use-one exception poses the application as voltage sensor for high-voltage power lines [20]. The size of these instruments $(10 \mathrm{~cm}$ for the crystal alone), however, impedes field mapping inside beam shaping elements.

The only 3D mapping of an electrostatic field was achieved with the force-field microscope of reference [21]. This elaborated approach exploits an optically trapped charged microsphere which can be positioned to scan the electric field in a volume. While this method excels for small volumes $\left(1000 \mu \mathrm{m}^{3}\right)$, it is hardly practical for mapping the field of macroscopic components.

Recently, a transduction method was introduced, enabling field mapping in meso and macroscopic volumes $[22,23]$. It exploits the electrostatic induction occurring inside a conducting body (here, doped silicon). The charged surfaces of the polarized conductor are, due to the external field $\mathbf{E}$, subject to an electrostatic force $\mathbf{F}_{\mathrm{es}}=Q \mathbf{E}$, which causes a deflection in a microelectromechanical system (MEMS). Here, $Q$ is the induced charge on one side of the conductor, the whole body remains neutral. Since $Q$ depends linearly on $\mathbf{E}, \mathbf{F}_{\mathrm{es}} \propto \mathbf{E}^{2}$. The deflection is read out with an on-chip optical shutter. With this kind of readout, remarkable sensitivities of below $1 \mathrm{pm} / \sqrt{\mathrm{Hz}}$ has been achieved $[24,25]$.

Here we describe a sensor probe (volume $<1.5 \mathrm{~cm}^{3}$ ) based on this transduction and use it to characterize the fields generated by the EQP. Since the quadrupole field is inhomogeneous, the suitability of the MEMS and its spatial resolution were investigated by finite element method (FEM) simulations. A sketch of the FODO with one of

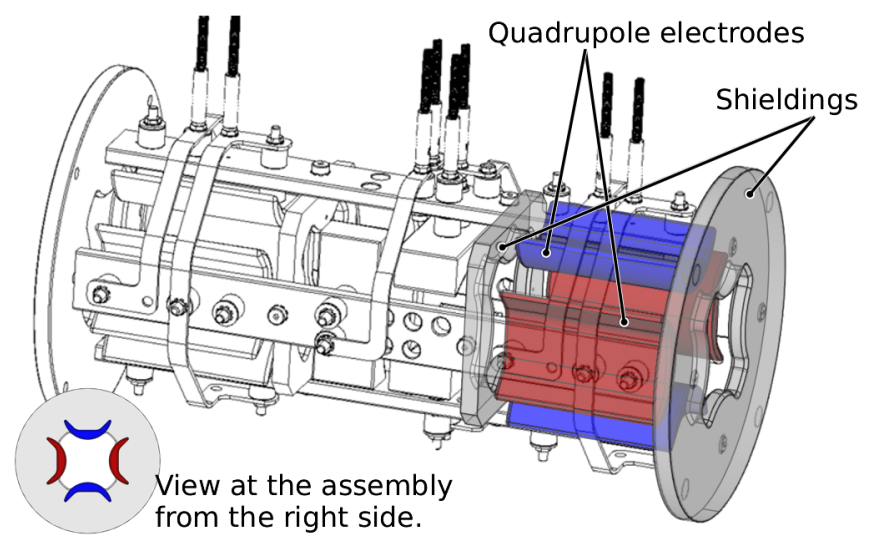

FIG. 1. Sketch of the FODO assembly. Elements of the simplified model are highlighted. Different colors represent the applied voltage at the poles: red (+) and blue (-). Frontal and rearward shielding plates for mitigating of the fringe field influence are highlighted in grey.

the EQPs highlighted is given in Fig. 1. The highlighted part was also used as underlying geometry for the simulations.

Aim of the simulations was to estimate the electrostatic force occurring in the MEMS chip (sized $6 \times 6 \times 0.9 \mathrm{~mm}^{3}$ ) and how the corresponding field deviates from the actual field at the same point. They also offered an insight into the spatial resolution of the sensor. Note that the MEMS is designed to deflect in only one direction and, thus, is selective only to the corresponding field component. In a first calibration simulation, the relation between force and field strength, $\mathbf{F}_{\mathrm{es}}=\alpha \mathbf{E}^{2}$, i.e., the coefficient $\alpha$, was determined. A value of $\alpha=1.29 \times 10^{-14} \mathrm{~N} /(\mathrm{V} / \mathrm{m})^{2}$ was obtained inside a uniform field ranging from 0 to $83.3 \mathrm{kV} / \mathrm{m}$ (see Supplemental Material [26]).

The MEMS was then placed in the volume between the EQP electrodes. It was moved to various positions within this volume to obtain the local force. Figure 2(a) shows the results for the center $y z$ plane cutting through the EQP. In these simulations, the potentials of the electrodes were set to $\pm 200 \mathrm{~V}$ and to $0 \mathrm{~V}$ for the shielding plates. This is much smaller than the typical voltages applied in the transfer lines $(< \pm 6 \mathrm{keV})$, but equals the voltage applied during the measurements. Both $y$ and $z$ components of the force have been obtained by appropriate orientation of the chip and were combined to obtain an $E$ field as $E=\sqrt{\left(F_{\mathrm{y}}+F_{\mathrm{z}}\right) / \alpha}$. The $x$ component has not been investigated, since it is nonzero only at the entrance and exit of the EQP and its net effect on the particle beam is negligible. Note that since $F_{\text {es }} \propto E^{2}, E$ corresponds to the absolute value of the field. However, since in this setting the polarity of the electrodes is known, so is the direction of $\mathbf{E}$.

The spatial resolution of the probe was investigated by moving the MEMS geometry along a line through the EQP field with a step size of $1 \mathrm{~mm}$ which is much smaller than the chip. The line was chosen to be between the points 


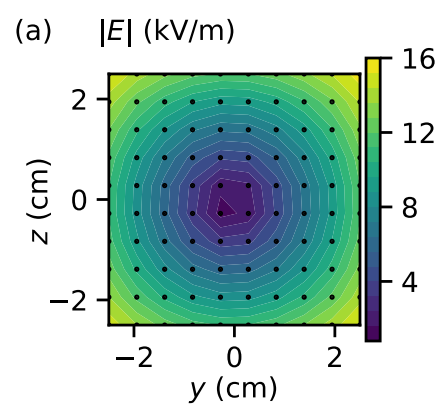

(b) Potential (kV)

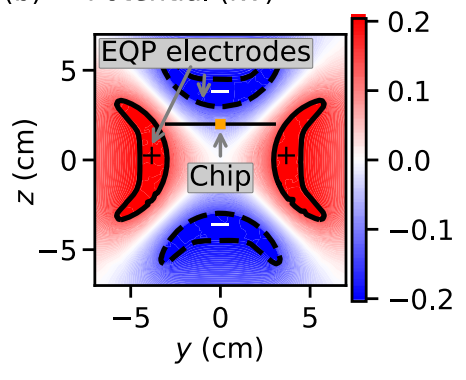

(d) $F_{\mathrm{y}}(\mu \mathrm{N})$

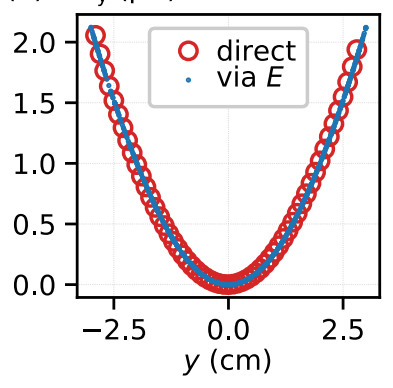

FIG. 2. (a) Cross section of the $E$ field recalculated from $F_{\mathrm{y}}$ and $F_{\mathrm{z}}$ obtained on the $y z$ plane in the EQP center. The black dots denote the positions of the MEMS geometry. A linear interpolation has been applied between them. (b) Potential at the cross section. For investigating the spatial resolution, the MEMS was moved along the horizontal line in $1 \mathrm{~mm}$ steps. (c) Field components obtained along the line. (d) Force obtained in the simulations (red circles) compared to the force calculated from the data in (c) by $F_{\mathrm{y}}=\alpha E_{\mathrm{y}}^{2}$. $(x, y, z)=(0,-3,2) \mathrm{cm}$ and $(0,3,2) \mathrm{cm}$ [see Fig. 2(b)]. Thus, only the $y$ component of the $E$ field is nonconstant [Fig. 2(c)]. Figure 2(d) shows that for the $y$ component, the directly obtained force matches the recalculated one exactly. Thus, the spatial resolution is in this case well below the width of the chip.

The situation is different for fields with higher curvature. Another analysis regarding the spatial resolution was performed in the field between a thin electrode and a plate electrode (see Supplemental Material Fig. S4 [26]). In this case, the results deviate strongly. However, since no such features are present in the EQP assembly, the spatial resolution of the probe can be expected to be sufficient.

In order to probe the EQP field, the MEMS chip is encapsulated within a 3D-printed acrylic holder and connected via optical fibers to the readout electronics placed at a remote location. Except from the Si chip, only dielectric materials are used in the probe to minimize field distortions. The light is guided through the chip and reflected by a right-angle prism and fed back to the fiber. Figure 3(a) depicts the fully assembled measurement setup. Linear stages are used to position the sensing tip. The components $E_{\mathrm{y}}$ and $E_{\mathrm{z}}$ were obtained by according orientation of the chip. For $E_{\mathrm{x}}$ however, one would need to probe the EQP through the tank, which is not possible with this setup.

The EQP was supplied with a sinusoidal voltage of $\pm 200 \mathrm{~V}$ and $f=150 \mathrm{~Hz}$ using a high-voltage amplifier (Tabor Electronics 9200A) controlled by a waveform

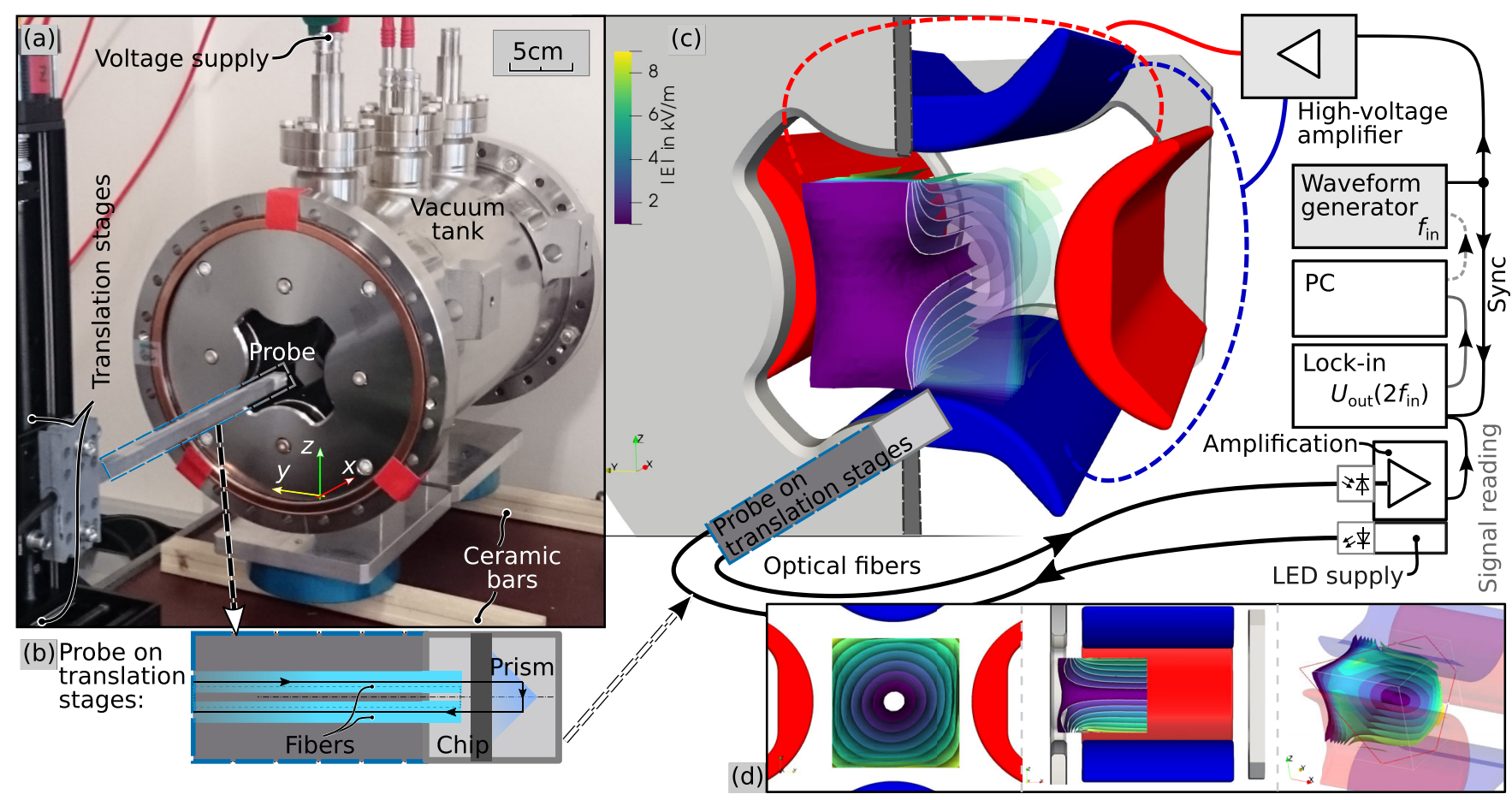

FIG. 3. (a) Setup for the EQP field characterization. (b) The zoom highlights the light path through the probe tip. (c) Schematic of the measurement and map of the field strength in the front half of the EQP. The data points were interpolated and represented as isosurfaces. (d) Different views on the visualized measurement data. 
generator. A low-frequency ac supply was chosen over a dc supply to use the lock-in method for the sake of improved results. Furthermore, this way it was possible to excite the mechanical resonance $\left(f_{0}=300 \mathrm{~Hz}\right)$ of the MEMS.

The probe was calibrated for both $y$ and $z$ direction in a uniform field provided by parallel plate electrodes. Voltages ranging from $500 \mathrm{mV}$ to $200 \mathrm{~V}$ were applied to the plates, which were $3.4 \mathrm{~cm}$ apart. This amounts to a field range of 7.4-5900 V/m. The responsivities of $R_{\mathrm{y}}=1.12 \times 10^{-7} \mathrm{~V}_{\text {rms }} /(\mathrm{V} / \mathrm{m})^{2}$ and $R_{\mathrm{z}}=0.74 \times$ $10^{-7} \mathrm{~V}_{\mathrm{rms}} /(\mathrm{V} / \mathrm{m})^{2}$ for $y$ and $z$ directions, respectively, were obtained with a least-squares fit. The difference of roughly $34 \%$ can be attributed to slightly different optical pathways. The field resolution limit was estimated from the noise spectrum of the probe (Supplemental Material Fig. S7 [26]). The zero-field output voltage of roughly $0.5 \mathrm{~m} \mathrm{~V}$ rms corresponds to a worst-case ( $z$ direction) field strength of $98 \mathrm{~V} / \mathrm{m}$. Taking into account the bandwidth $(2.6 \mathrm{~Hz})$ of the lock-in amplifier, this equals a resolution of $61 \mathrm{~V} / \mathrm{m} / \sqrt{\mathrm{Hz}}$.

Then, the 3D volume from the center plane to the outer edge of the shielding plate of the EQP was studied. It was $8 \times 5 \times 5 \mathrm{~cm}^{3}$ in size and with a step size of $2 \mathrm{~mm}$ in each direction, a total of $39 \times 25 \times 25=24375$ points were scanned. Figure 3(c) shows the obtained map of the field amplitude $|\mathbf{E}|$ composed of $E_{\mathrm{y}}$ and $E_{\mathrm{z}}$.

The probe was then used to map the most ideal field in the center cross section to compare it to the FEM simulations. Figures 4(a) and 4(b) show the corresponding results for output voltage and field strength of $y$ and $z$ components. Due to the different responsivities, the shape of the total output voltage appeared stretched, which was rectified by the probe calibration.

To confirm the spatial resolution predicted by the numerical analysis, the field along the same line as in Fig. 2(b) is recorded with a step size of $100 \mu \mathrm{m}$. The resulting output voltage and field strength for the $y$ direction are shown in Figs. 4(c) and 4(d). The field strength was again recalculated from the output voltages with the responsivities obtained during the calibrations. The spatial resolution for the linearly changing field component is qualitatively and quantitatively in good agreement to the prediction. The deviation from the simulated data is in the order of $3 \%$ which can be attributed to the makeshift holders for the fibers and probe tip.

It can be concluded that the EQP behaves like the simulations predict, which is one of the major benefits of electrostatic components. However, unforeseen effects can occur if the fabricated geometry deviates from the design. This is why tolerances of $\delta= \pm 50 \mu \mathrm{m}$ are required for the individual components. According to Ref. [27], this leads to a maximum transverse offset of one electrode of $\delta_{\max }=150 \mu \mathrm{m}$ and a mean cumulative offset of $\bar{\delta}=100 \mu \mathrm{m}$.

Assuming a quadrupole composed of four point charges, an analytical perturbation estimation of the field can be
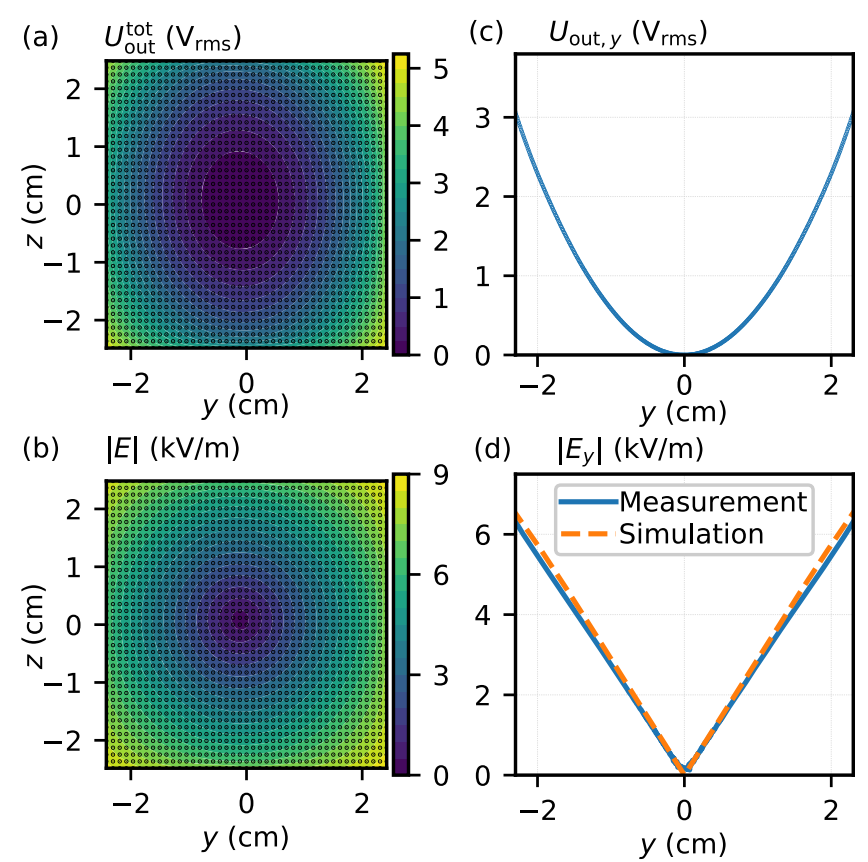

(d) $\left|E_{y}\right|(\mathrm{kV} / \mathrm{m})$

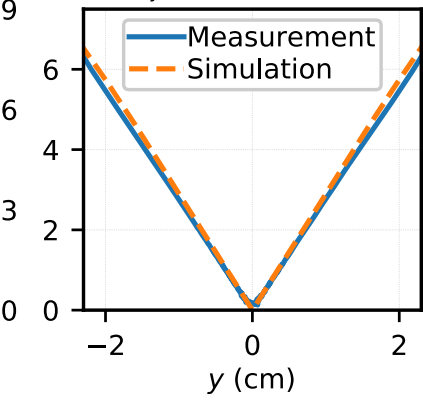

FIG. 4. (a) Output voltage $U_{\text {tot }}=U_{\mathrm{y}}+U_{\mathrm{z}}$ from the EQP center cross section. (b) Corresponding field strength $|E|$ obtained with the respective responsivities $R_{\mathrm{y}, \mathrm{z}}$. The black dots denote the positions of the probe. The data were linearly interpolated between them. (c) Spatial resolution of the $E$ field probe in terms of recorded voltage. The MEMS was moved along a line as in Fig. 2(b) with a step size of $100 \mu \mathrm{m}$. (d) $\left|E_{\mathrm{y}}\right|$ recalculated from the data in (c) compared to the simulations.

performed for a small spatial offset of one of the charges. For the point charges $+q$ located on the $y$ axis at a distance $\pm s$ from the origin and the charges $-q$ on the $z$ axis at a distance $\pm s$ from the origin, the perturbed potential for a displacement $\Delta y=\delta$ of the charge at $y=-s$ is given as

$$
\Phi \approx \Phi_{0}-\frac{q}{4 \pi \varepsilon_{0}} \frac{\delta}{s}\left(\frac{1}{s}-\frac{2 y}{s^{2}}+\frac{3}{s^{3}}\left(y^{2}-\frac{z^{2}}{2}\right)\right),
$$

where $\varepsilon_{0}$ is the vacuum permittivity and $\Phi_{0}$ the unperturbed potential. The perturbed potential also contains a monopole and a dipole term. Higher poles and higher order $\delta$ terms were omitted. From that, the field components $E_{i}=$ $-\partial \Phi / \partial x_{i}$ can be calculated. Thus, with $U_{\text {out }} \propto E^{2}$, the relative change of the corresponding sensor output voltages can be written as

$$
\frac{U_{\text {out }, \mathrm{y}}}{U_{\mathrm{out}, \mathrm{y}}^{0}} \approx 1+2 \frac{\delta}{S}\left(\frac{1}{3} \frac{s}{y}-1\right) \text { and } \quad \frac{U_{\mathrm{out}, \mathrm{z}}}{U_{\mathrm{out}, \mathrm{z}}^{0}} \approx 1-\frac{\delta}{S} .
$$

The poles can be viewed as equipotential surfaces and approximate the hyperbolic shape of the quadrupole potential. The radial position $s$ of the imaginary point charges is, thus, given by $s=\sqrt{2} r_{0}$, with $r_{0}=3 \mathrm{~cm}$ being the distance of the poles from the coordinate origin. 


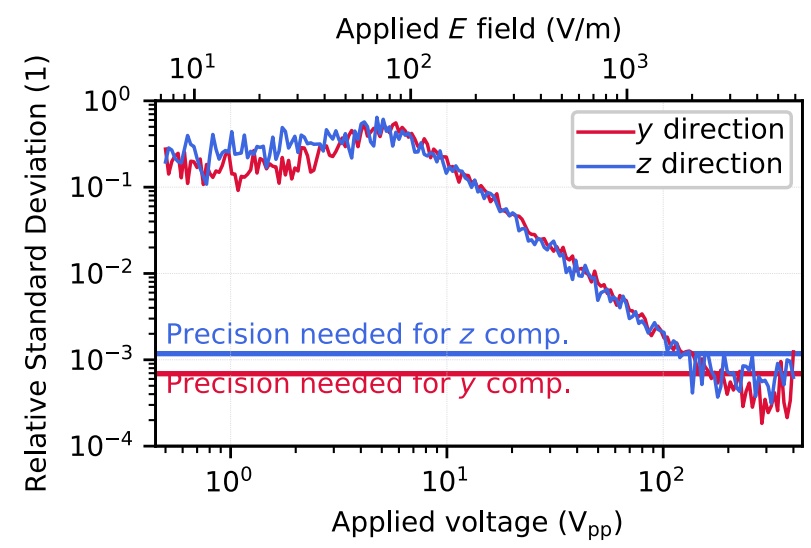

FIG. 5. Measurement precision as function of the input $E$ field. The horizontal lines denote the precision necessary to resolve the perturbation due to a transverse offset of one electrode by the fabrication tolerances. Below roughly $100 \mathrm{~V} / \mathrm{m}$ the curves are flat due to the noise floor of the sensor. Then the relative standard deviation decreases in the same way as the applied field increases. Above $2 \mathrm{kV} / \mathrm{m}$, the curves become flatter again, pointing out the precision limit of the current sensor and setup.

This leads to deviations in $U_{\text {out }, i}$ of $\pm 0.07 \%$ (with $y=2 \mathrm{~cm}$ ) and $\pm 0.12 \%$ for $y$ and $z$ components, respectively.

The precision of a single measurement with the MEMS probe is given by the standard deviation relative to the measured signal. For being able to check if the tolerances are met, the precision needs to be smaller than the above derived deviations. The standard deviation for the probe was determined during the calibration (see Supplemental Material Fig. S6 [26]). Figure 5 shows that the probe is able to resolve these tiny field perturbations, if the unperturbed field is large enough $(\gtrsim 2 \mathrm{kV} / \mathrm{m})$.

We presented a way to scan the electric field strength in a $3 \mathrm{D}$ volume. It has been shown that remarkable spatial resolution can be achieved for fields with sufficiently low curvature. The field of an electrostatic quadrupole was mapped and is used for low-energy antimatter beam steering and control in the ELENA experiment at CERN. The results confirmed experimentally that such electrostatic components behave like numerical analyses predict. However, this reliability hinges on tight fabrication tolerances and flawless production. Future projects envisaged at CERN involve electrostatic elements in a ring, where due to the beam passing the element multiple times, the required field homogeneity is tighter than for the abovementioned EQPs. For the ongoing study of an electrostatic ring to measure the electric dipole moment [28-32], a field homogeneity of $10^{-4}$ is required that corresponds to mechanical fabrication tolerances of a few micrometers. While the requirements of the ELENA transfer line EQP [tolerance of $\mathcal{O}(100 \mu \mathrm{m})$ ] are still feasible, it will be difficult to cost-effectively produce electrostatic ring elements. Here, a precise field measurement of the series production would enable for mitigation measures such as sorting elements and active compensation.

Apart from accelerators and particle storage rings, such a potentially low-cost probe can also be of interest for power infrastructure, atmospheric electricity research or worker safeguarding.

In future works, sensor elements will be combined to measure all three field axes at once. Also commonly used spatial averaging for magnetic field mapping, handled by so-called quad Hall plates [33], could be adapted for our needs. In an ideal case, the effect of the spatial offset should be completely eliminated utilizing this technique.

The mounting of the sensor needs upgrading to avoid bending, tilt, and misalignment of the chip. Furthermore, improving the optical paths and coupling is expected to lead to a significantly increased signal-to-noise ratio and lower power consumption. This will also be achieved by integration of the current makeshift electronics. We expect that the extreme precision necessary for sorting out electrostatic ring elements will be reached with these improvements.

This work was supported financially by the Austrian Science Fund (FWF, Research Grant No. P28404-NBL), the European Regional Development Fund (ERDF), and the Province of Lower Austria. The data supporting the plots within this Letter and other findings of this study are available from the corresponding author upon reasonable request.

*Corresponding author. andreas.kainz@tuwien.ac.at

[1] G. E. Fischer, Iron dominated magnets, Technical Report No. SLAC-PUB-3726, Stanford Linear Accelerator Center, Stanford University, Stanford, CA, USA, 1985.

[2] A. Noda, M. Yoshizawa, M. Mutou, and T. Fujino, Measuring system of multipole fields of the quadrupole magnet in drift-tubes of a linear accelerator, Technical Report No. INS-NUMA-32, University of Tokyo, Japan, 1982.

[3] J. L. Conradie, D. T. Fourie, J. C. Cornell, and G. C. W. Lloyd, in Proceedings of the Eleventh International Conference on Cyclotrons and their Applications (CERN, Tokyo, Japan, 1986), pp. 321-324.

[4] M. Stifter, H. Steiner, W. Hortschitz, T. Sauter, T. Glatzl, A. Dabsch, and F. Keplinger, IEEE Sens. J. 16, 8744 (2016).

[5] C. P. Welsch and H. Danared, in Proceedings of EPAC 2006 (EPAC, Edinburgh, Scotland, 2006).

[6] E. Widmann, in 9th International Conference on Nuclear Physics at Storage Rings (IOP, Sankt Goar, Germany, 2015) arXiv:1502.03687.

[7] V. Chohan et al., Extra low energy antiproton (ELENA) ring and its transfer lines, Technical Report No. CERN-2014002, CERN, 2014.

[8] S. Maury, W. Oelert, W. Bartmann, P. Belochitskii, H. Breuker, F. Butin, C. Carli, T. Eriksson, S. Pasinelli, and G. Tranquille, Hyperfine Interact. 229, 105 (2014). 
[9] W. Bartmann, P. Belochitskii, H. Breuker, F. Butin, C. Carli, T. Eriksson, W. Oelert, R. Ostojic, S. Pasinelli, and G. Tranquille, Philos. Trans. R. Soc., A 376, 20170266 (2018).

[10] T. Eriksson, W. Bartmann, P. Belochitskii, L. Bojtar, H. Breuker, F. Butin, C. Carli, B. Dupuy, P. Freyermuth, L. Joergensen, B. Lefort, J. Mertens, R. Ostojic, S. Pasinelli, G. Tranquille, G. W. Oelert, and J. G. University, in Proceedings of IPAC2017 (JACoW, Copenhagen, Denmark, 2017), http://accelconf.web.cern.ch/AccelConf/ipac2017/doi/ JACoW-IPAC2017-WEPVA034.html.

[11] D. A. Hill and M. Kanda, The Measurement, Instrumentation, and Sensors Handbook XXV, Section 47, Electric Field Strength (CRC Press LLC and IEEE Press, Boca Raton, Florida, 1999).

[12] M. N. Horenstein and P. R. Stone, J. Electrost. 51-52, 515 (2001).

[13] H. Kirkham, in Conference on Precision Electromagnetic Measurements (IEEE, Ottawa, 2002).

[14] B. Bahreyni, G. Wijeweera, C. Shafai, and A. Rajapakse, J. Microelectromech. Syst. 17, 31 (2008).

[15] S. Ghionea, D. Hull, and K. Williams, J. Electrost. 71, 1076 (2013).

[16] D. Chen, J. Zhao, Y. Wang, Z. Xu, and J. Xie, Appl. Phys. Lett. 112, 013502 (2018).

[17] A. Rogers, Opt. Laser Technol. 9, 273 (1977).

[18] A. H. Rose, S. M. Etzel, and K. B. Rochford, J. Lightwave Technol. 17, 1042 (1999).

[19] R. Zeng, B. Wang, B. Niu, and Z. Yu, Sensors 12, 11406 (2012).

[20] K. Bohnert, P. Gabus, H. Brändle, and A. Khan, in Proceedings of the 16th International Conference on Optical Fiber Sensors, Nara, Japan (Institute of Electronics, Information and Communication Engineers, 2003), Vol. 1317.
[21] C. P. Blakemore, A. D. Rider, S. Roy, Q. Wang, A. Kawasaki, and G. Gratta, Phys. Rev. A 99, 023816 (2019).

[22] A. Kainz, H. Steiner, J. Schalko, A. Jachimowicz, F. Kohl, M. Stifter, R. Beigelbeck, F. Keplinger, and W. Hortschitz, Nat. Electron. 1, 68 (2018).

[23] A. Kainz, W. Hortschitz, H. Steiner, M. Stifter, J. Schalko, A. Jachimowicz, and F. Keplinger, Appl. Phys. Lett. 113, 143505 (2018).

[24] W. Hortschitz, A. Kainz, H. Steiner, M. Stifter, F. Kohl, J. Schalko, T. Sauter, and F. Keplinger, Procedia Eng. 87, 835 (2014).

[25] R. Middlemiss, A. Samarelli, D. Paul, J. Hough, S. Rowan, and G. Hammond, Nature (London) 531, 614 (2016).

[26] See Supplemental Material at http://link.aps.org/ supplemental/10.1103/PhysRevLett.122.244801 for additional information about the MEMS, modeling and measurements.

[27] D. Barna, W. Bartmann, and R. Ostojic, Electrostatic quadrupole assembly (ZQNA) for elena transfer lines, engineering specification 1410617 (CERN, 2014), eDMS_CERN's Engineering Data Management Service.

[28] Feasibility study for an EDM storage ring, https://indico .cern.ch/event/765096/contributions/3295545/.

[29] D. Eversmann et al. (JEDI Collaboration), Phys. Rev. Lett. 115, 094801 (2015).

[30] V. Anastassopoulos et al., Rev. Sci. Instrum. 87, 115116 (2016).

[31] N. Hempelmann et al. (JEDI Collaboration), Phys. Rev. Lett. 119, 014801 (2017).

[32] V. Andreev and N. Hutzler, Nature (London) 562, 355 (2018).

[33] R. Popovic, Hall Effect Devices, Section 5.6, Incorporating a Hall Device into a Circuit (Institute of Physics Publishing, Bristol and Philadelphia, 2004). 